\title{
Dominant channels of exciton spin relaxation in photoexcited self-assembled (In,Ga)As quantum dots
}

\author{
Yu-Huai Liao, ${ }^{1}$ Juan I. Climente, ${ }^{2}$ and Shun-Jen Cheng ${ }^{1, *}$ \\ ${ }^{1}$ Department of Electrophysics, National Chiao Tung University, Hsinchu 300, Taiwan, Republic of China \\ ${ }^{2}$ Departament de Química Física i Analítica, Universitat Jaume I, Castello E-12080, Spain \\ (Received 15 October 2010; revised manuscript received 2 March 2011; published 22 April 2011)
}

\begin{abstract}
We present a comprehensive theoretical investigation of spin relaxation processes of excitons in photoexcited self-assembled quantum dots. The exciton spin relaxations are considered between dark- and bright-exciton states via the channels created by various spin-admixture mechanisms, including electron Rashba and Dresselhaus spinorbital couplings (SOCs), hole linear and hole cubic SOCs, and electron hyperfine interactions, incorporated with single- and double-phonon processes. The hole-Dresselhaus SOC is identified as the dominant spin-admixture mechanism, leading to relaxation rates as fast as $\sim 10^{-2} \mathrm{~ns}^{-1}$, consistent with recent observations. Moreover, due to significant electron-hole exchange interactions, single-phonon processes are unusually dominant over two-phonon ones in a photoexcited dot even at temperatures as high as $15 \mathrm{~K}$.
\end{abstract}

DOI: 10.1103/PhysRevB.83.165317

PACS number(s): 78.67.Hc, 71.35.Cc, 71.70.Ej, 78.47.-p

\section{INTRODUCTION}

Spin dynamics in semiconductor quantum dots (QDs) is a subject of interest in the current endeavor to develop spintronics and quantum information processing applications. ${ }^{1}$ It has been widely believed for a long time that the discrete nature of QDs can make the spin relaxation times of confined carriers long enough for further applications. ${ }^{2}$ Indeed, the spin lifetimes of electrons confined in QDs have been experimentally confirmed to reach up to $1 s .^{3}$ Based on such long-lived electron spins, coherently controlled quantum gate devices made of electrode-defined QDs have been recently realized. ${ }^{4}$

In quantum photonic applications, InGaAs self-assembled QDs have been recently demonstrated as useful quantum light sources used in photonic quantum teleportation and cryptography. ${ }^{5}$ The generation rate of single-photon emission from the dots is, however, severely limited by the undesired fast spin relaxation of excitons, ${ }^{6,7}$ reported to be as fast as $\sim 10^{2}$ ns in recent experiments. ${ }^{8}$ Such transitions mainly occur between bright-exciton (BX) and dark-exciton (DX) states split by the $e-h$ exchange interaction, which is of hundreds of $\mu \mathrm{eV} .{ }^{9}$ What is more, the DX-to-BX transitions have been shown to be ultimately responsible for spin transitions within the BX doublet, ${ }^{10}$ so that they also limit the performance of entangled photon pair generators.

By contrast to existing extensive research for electrons or holes in QDs, ${ }^{1}$ the fundamental understandings of the spin relaxation processes of excitons in QDs are still incomplete. As a two-component quasiparticle, the spin dynamical processes of a quantum-confined exciton involve more complications, mixing various spin-flip mechanisms and phonon processes via the intrinsic $e-h$ mutual interactions. To date, only the intrinsic mixing of heavy- and light-hole states ${ }^{11}$ and the linear-in- $p$ spin-orbit coupling (SOC) of valence band holes ${ }^{12}$ have been theoretically studied as possible exciton spin-flip mechanisms, which, however, predict spin relaxation rates far below the observed values. ${ }^{8}$

In this work, we attempt to fill the gap between existing experiments and theoretical predictions. We present a comprehensive investigation of spin relaxation of single excitons in InGaAs self-assembled QDs using both an analytical method and a numerical exact diagonalization technique, with a full consideration of $e-h$ exchange interactions, all possible electron and hole SOCs, hyperfine interactions, and particlephonon couplings in single- and two-phonon processes. We explain the fast exciton spin relaxation observed in QDs in terms of pronounced hole-Dresselhaus SOCs and $e$ - $h$ exchange interactions in predominant single-phonon processes.

\section{THEORY}

\section{A. Model and Hamiltonian}

We start with an interacting Hamiltonian for a single neutral exciton confined in a phonon-free quantum dot:

$$
H_{X}^{0}=H_{e}+H_{h}+V_{e h}+V_{e h}^{x c}+H_{\mathrm{SO}}^{e}+H_{\mathrm{SO}}^{h} .
$$

Here $H_{j}$ denotes the noninteracting single-electron $(j=$ $e$ ) or single-hole $(j=h)$ Hamiltonian in a parabolic QD: $H_{j}=\frac{\mathbf{p}_{j}^{2}}{2 m_{j}}+\frac{1}{2} m_{j} \omega_{j}^{2} r_{j, \|}^{2}+V_{j}\left(z_{j}\right)$, where $\mathbf{p}_{j}$ are the operators of linear momentum, $m_{j}$ are the effective masses of particles, $\omega_{j}$ parametrizes the lateral confining potential, $V_{j}\left(z_{j}\right)$ is the vertical square confining potential of thickness $d_{z}$, and $\mathbf{r}_{j, \|}=\left(x_{j}, y_{j}\right)$ is the in-plane coordinate. Within the model, the single-particle wave function can be written in a separable form: $\Psi_{n_{\|}, n_{z}}^{j}\left(x_{j}, y_{j}, z_{j}\right)=$ $\psi_{n_{\|}}\left(\mathbf{r}_{j, \|}\right) g_{n_{z}}\left(z_{j}\right)$. The in-plane part of $H_{j}$ yields the explicit two-dimensional (2D) Fock-Darwin (FD) energy spectrum $\varepsilon_{s}^{j}=\hbar \omega_{j}, \varepsilon_{p^{ \pm}}^{j}=2 \hbar \omega_{j}, \ldots$, and the single-particle wave functions $\psi_{s}\left(\mathbf{r}_{j, \|}\right)=\frac{1}{l_{0} \sqrt{\pi}} \exp \left(-r_{j, \|}^{2} / 2 l_{0}^{2}\right), \psi_{p^{ \pm}}\left(\mathbf{r}_{j, \|}\right)=$ $\frac{r_{j, \|}}{l_{0}^{2} \sqrt{\pi}} \exp \left(-r_{j, \|}^{2} / 2 l_{0}^{2}\right) \times e^{ \pm i \phi}, \ldots,{ }^{13}$ where the subscripts $s, p^{+}$, and $p^{-}$indicate the atomiclike $s$ - and $p$-shell orbitals of QD with orbital angular momentum projection $L_{z}=0,+1$, and -1 , respectively, and $l_{0} \equiv \sqrt{\hbar / m_{e} \omega_{e}}=\sqrt{\hbar / m_{h} \omega_{h}}$ is the characteristic lateral extent of wave functions (as depicted by the lower-right schematics in Fig. 1).

The terms $V_{e h}$ and $V_{e h}^{x c}$ are the $e$-h Coulomb direct and exchange interactions, respectively. It is mainly the attractive direct interaction $V_{e h}$ making an $e$ - $h$ pair bind together to form an exciton. $V_{e h}$ does not, however, affect the spin structure of exciton states, and we shall treat it as a constant offset 


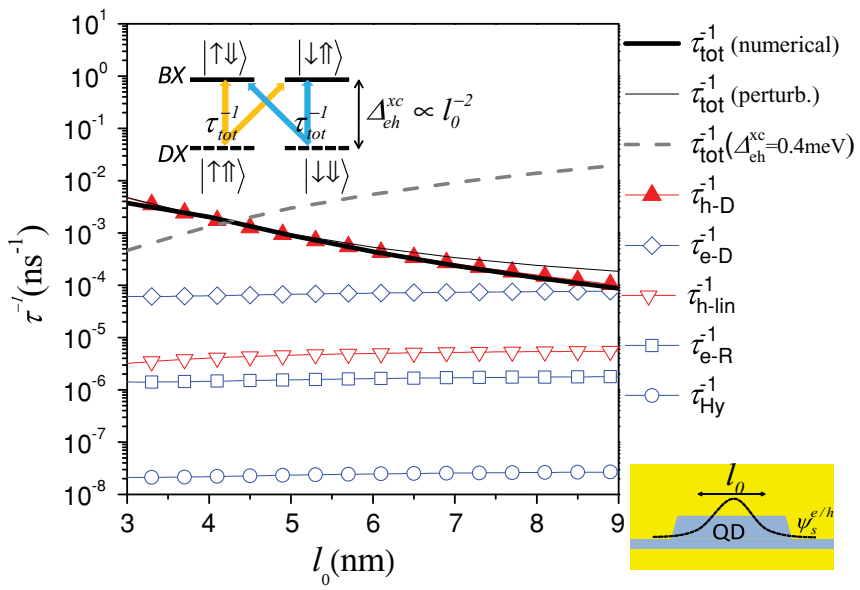

FIG. 1. (Color online) Exciton spin relaxation rates in the DX to $\mathrm{BX}$ transition and the rates contributed from each spin-flip mechanism, as functions of the QD size. Results with fixed $\Delta_{e h}^{x c}=$ $0.4 \mathrm{meV}$ (dashed line) are also presented for comparison. Inset: Schematic of the DX to BX conversion channels.

of energy. The fourfold spin degenerate single-exciton states are split by $e-h$ exchange interaction $V_{e h}^{x c}$ into a lower-energy optically inactive doublet with $M_{z}=s_{z}+j_{z}= \pm 2$ (the so-called DX states $\left|s_{z}=+\frac{1}{2}, j_{z}=+\frac{3}{2}\right\rangle=|\uparrow \uparrow\rangle \quad$ and $\left.\left|s_{z}=-\frac{1}{2}, j_{z}=-\frac{3}{2}\right\rangle=|\downarrow \Downarrow\rangle\right)$ and a higher-energy doublet with $M_{z}= \pm 1$ (the BX states $|\uparrow \Downarrow\rangle$ and $|\downarrow \Uparrow\rangle$ ) with an energy separation $\Delta_{e h}^{x c} \sim 10^{2}-10^{3} \mu \mathrm{eV} . V_{e h}^{x c}$ thus acts as an effective field coupled to the exciton spin but, unlike an externally applied field, itself is associated with the dot structure. Treating the short-range part of the corresponding $e$ - $h$ exchange interaction as the dominant component, the BX-DX splitting $\Delta_{e h}^{x c}$ for the low-lying exciton states can be modeled as ${ }^{14}$

$\Delta_{e h}^{x c}=\Delta_{e h, \text { bulk }}^{x c} \times\left(\pi a_{B}^{* 3}\right) \int d^{3} r\left|\Psi_{s, 0}^{e}(\mathbf{r}) \Psi_{s, 0}^{h}(\mathbf{r})\right|^{2} \propto l_{0}^{-2}$,

where $\Delta_{e h \text {,bulk }}^{x c}$ is the bulk $e$ - $h$ exchange energy and $a_{B}^{*}$ the effective exciton Bohr radius for InGaAs. Note that the smaller the dot, the larger the $\Delta_{e h}^{x c}$.

The term $H_{\mathrm{SO}}^{e}\left(H_{\mathrm{SO}}^{h}\right)$ denotes the electron (hole) SOC. For electrons, the SOC Hamiltonian consists of the Rashba and Dresselhaus terms, $H_{\mathrm{SO}}^{e}=H_{R}^{e}+H_{D}^{e}{ }^{1}$ being

$$
\begin{aligned}
& H_{R}^{e}=\left(\alpha_{e} / i \hbar\right)\left(p_{+} \sigma_{-}-p_{-} \sigma_{+}\right), \\
& H_{D}^{e}=-\left(\beta_{e} / \hbar\right)\left(p_{+} \sigma_{+}+p_{-} \sigma_{-}\right),
\end{aligned}
$$

where $p_{ \pm} \equiv p_{x} \pm i p_{y} \quad\left(\sigma_{ \pm} \equiv \sigma_{x} \pm i \sigma_{y}\right)$ are orbital (spin) ladder operators for $(s=1 / 2)$ electron and $\alpha_{e}\left(\beta_{e}\right)$ is the $e$-Rashba (Dresselhaus) SOC constant.

For holes, we consider the $h$-SOC Hamiltonian as $H_{S O}^{h}=$ $H_{l i n}^{h}+H_{D}^{h}$ consisting of the relevant $p$-linear and -cubic terms: ${ }^{12,15}$

$$
\begin{gathered}
H_{l i n}^{h}=-\left(\alpha_{h} / \hbar\right)\left(p_{+} \sigma_{-}+p_{-} \sigma_{+}\right), \\
H_{D}^{h}=-\left(\beta_{h} / \hbar^{3}\right)\left(p_{+} p_{-} p_{+} \sigma_{-}+p_{-} p_{+} p_{-} \sigma_{+}\right),
\end{gathered}
$$

where $\alpha_{h}\left(\beta_{h}\right)$ is the $p$-linear (-cubic $h$-Dresselhaus) SOC constant. Equations (5) and (6) are expressed in terms of Pauli operators for the pseudospin of heavy holes defined by the spin replacement $j=3 / 2 \rightarrow s_{h}=1 / 2$ and $j_{z}= \pm 3 / 2 \rightarrow$ $s_{z}^{h}= \pm 1 / 2$. For brevity, the superscripts $h$ for the hole spin operators are removed. The $h$-Rashba SOC is irrelevant in the problem here since $H_{h-R} \propto p_{ \pm}^{3}$ involves only the remote $h$ states above the $d$ shell. ${ }^{15}$

The eigenenergies and eigenstates of a spin exciton in a phonon-free QD can be numerically obtained by solving the eigenequation $H_{X}^{0}|X ; i\rangle=E_{X ; i}|X ; i\rangle$ using an exact diagonalization method for the matrix of $H_{X}^{0}$ in the basis of exciton configurations built up from the FD orbitals (with a typical number of FD orbitals $\approx 15$ and that of exciton configurations $\approx 900$ ). The parameters used in the calculations throughout this work are summarized in Table V in the Appendix.

\section{B. Relaxation rates}

Next, we consider the QD coupled to the acoustic phonon bath by introducing the particle-phonon couplings into the QD system, being

$$
H_{j-p h}=\sum_{\nu \mathbf{q}} M_{\nu}^{j}(\mathbf{q})\left(b_{\mathbf{q}} e^{i \mathbf{q} \cdot \mathbf{r}_{j}}+b_{\mathbf{q}}^{\dagger} e^{-i \mathbf{q} \cdot \mathbf{r}_{j}}\right) .
$$

Here $\mathbf{q}$ is the phonon wave vector, $v=L D, T D, L P, T P$ denote the kinds of phonon modes (longitudinal or transversal modes of deformation phonons or piezoelectricity phonons), $b_{\mathbf{q}}$ and $b_{\mathbf{q}}^{\dagger}$ are the phonon creation and annihilation operators, and $M_{v}^{j}$ are the phonon scattering matrix elements. Table I summarizes the expressions for $M_{\nu}^{j}(\mathbf{q})$ as functions of the phonon wave vector $\mathbf{q}=\left(q_{x}, q_{y}, q_{z}\right)$ and relevant material parameters.

The exciton spin relaxation rate for the transition from DX states to BX ones involving single-phonon processes (as illustrated by the schematics in the inset of Fig. 1) are evaluated using Fermi's golden rule:

$$
\tau_{\text {tot }}^{-1}=\frac{2 \pi}{\hbar} \sum_{f} \sum_{j=e, h} \sum_{v \mathbf{q}}\left|\left\langle f\left|V_{\nu \mathbf{q}}^{j}\right| i\right\rangle\right|^{2} \delta\left(E_{f i}-\hbar \omega_{\nu q}\right) N_{q_{v}} .
$$

\begin{tabular}{|c|c|c|c|c|}
\hline & $v=L D$ & $v=T D$ & $v=L P$ & $v=T P$ \\
\hline$\left|M_{v}^{j}(\mathbf{q})\right|^{2}$ & $\frac{\hbar D_{j, L D}^{2}}{2 d c_{L D} \Omega} q$ & $\frac{\hbar D_{j-T D}^{2}}{2 d c_{T D} \Omega} \frac{q_{z}^{2}\left(q_{x}^{2}+q_{y}^{2}\right)}{q^{3}}$ & $\frac{32 \pi^{2} \hbar e^{2} h_{14}^{2}}{\epsilon^{2} d c_{L P} \Omega} \frac{\left(3 q_{x} q_{y} q_{z}\right)^{2}}{q^{7}}$ & $\begin{array}{l}\frac{32 \pi^{2} \hbar e^{2} h_{14}^{2}}{\epsilon^{2} d c_{T P}} \mid \frac{\left(q_{x} q_{y}\right)^{2}+\left(q_{y} q_{z}\right)^{2}+\left(q_{z} q_{x}\right)^{2}}{q^{5}} \\
-\frac{\left(3 q_{x} q_{y} q_{z}\right)^{2}}{q^{7}} \mid\end{array}$ \\
\hline
\end{tabular}

TABLE I. Expressions for phonon scattering matrix elements $M_{v}^{j}$ as functions of the phonon wave vector $\mathbf{q}=\left(q_{x}, q_{y}, q_{z}\right)$ and phonon parameters for InGaAs. $v(=L D, T D, L P, T P)$ denotes the kinds of phonon modes (longitudinal or transversal modes of deformation phonons or piezoelectricity phonons). $\Omega$ denotes the crystal volume. Other symbols for phonon parameters are summarized in Table $V$ in the Appendix. 
Here $V_{\nu \mathbf{q}}^{j}=M_{\nu}^{j}(\mathbf{q}) e^{i \mathbf{q} \mathbf{r}_{j}}, \hbar \omega_{\nu q}$ is the phonon energy, and $|i\rangle$ and $|f\rangle$ are the initial (DX) and final (BX) states, respectively. $E_{f i} \equiv E_{f}-E_{i}$ is the energy difference between $|f\rangle$ and $|i\rangle$. The $\delta$ function in Eq. (8) ensures the resonance condition $\hbar \omega_{v q}=\left|E_{f i}\right|$ in the single-phonon processes, which indicates $\hbar c_{\nu} q_{\nu 0}=\Delta_{e h}^{x c} \propto q_{\nu 0}\left(c_{\nu}\right.$ is the speed of acoustic phonon). $N_{q_{v}}=1 /\left(e^{\hbar \omega_{v q} / k_{B} T}-1\right)$ is the phonon population, with $T$ being the temperature and $k_{B}$ the Boltzmann constant.

\section{NUMERICAL RESULTS}

\section{A. Single-phonon processes}

Figure 1 presents the numerically calculated total rates $\tau_{\text {tot }}^{-1}$ of single excitons in QDs of fixed thickness $d_{z}=3 \mathrm{~nm}$ but with varying lateral sizes. It can be seen that the total spin relaxation rate of the exciton is at the scale of $10^{-3}-10^{-2} \mathrm{~ns}^{-1}$ consistent with recent observations. ${ }^{8}$ For further insight, one can extract the individual contributions $\tau_{j-S O}^{-1}$ from each $j$ (electron or hole) SOC term in the total rate $\tau_{\text {tot }}^{-1}$. Among all spin mechanisms, the $p$-cubic $h$-Dresselhaus SOC leads to the fastest spin relaxation rate, $\tau_{h-D}^{-1} \sim 10^{-3}-10^{-2} \mathrm{~ns}^{-1}$. This is 1 order of magnitude higher than that of $e$-Dresselhaus $\operatorname{SOC}\left(\tau_{e-D}^{-1} \sim 10^{-4} \mathrm{~ns}^{-1}\right)$ for self-assembled dots with typical $l_{0}<6 \mathrm{~nm}$ and faster than those due to intrinsic heavy-hole (hh)-light-hole(lh) mixing in excitons. ${ }^{8,11}$ The linear hole SO interaction, which was proposed as the exciton spin relaxation mechanism in large QDs, ${ }^{12}$ is found to be a weak effect in selfassembled QDs, leading to a slow rate of $\tau_{h-\text { lin }}^{-1} \sim 10^{-6} \mathrm{~ns}^{-1}$.

For spin transitions mediated by the electron-nuclei hyperfine (Hy) interaction, an exact diagonalization procedure like that used for the SOCs is quite challenging because the number of involved nuclei is over millions. We thus separately evaluate the spin relaxation rate via the Hy interactions by using the perturbation method presented in Ref. [16]. The calculated Hy-interaction-mediated spin relaxation rate of exciton in a QD is quite slow, $\tau_{H y}^{-1} \sim 10^{-8} \mathrm{~ns}^{-1}$, incomparable to the spin relaxation rate arising from other SOCs.

A remarkable feature of Fig. 1 is that the spin relaxation of excitons, as opposed to that of single electrons, is not really suppressed by the reduced dot sizes. Instead, the size effect of QDs makes the spin relaxation rate even faster. This is because the smaller the QD, the stronger the Coulomb interaction and hence $\Delta_{e h}^{x c}$. Larger interlevel spacing translates into larger density of acoustic phonons, more efficient carrier-phonon coupling, ${ }^{17,18}$ and stronger spin-orbital admixture. Further understanding of this is provided by a perturbational analysis we carry out in Sec. IV.

\section{B. Two-phonon processes}

Next, we examine the influence of two-phonon processes on the spin relaxation of excitons. The examination of the two-phonon process effect is necessary here since there has been evidence that, in the absence of magnetic fields, the spin relaxation of holes is dominated by two-phonon processes starting from temperatures below $1 \mathrm{~K} .{ }^{19}$ We consider processes involving an initial absorption to a virtual state $|n\rangle$, followed by an emission to the final state, as illustrated in the inset of Fig. 2(a). The main spin-admixture mechanism is hole-
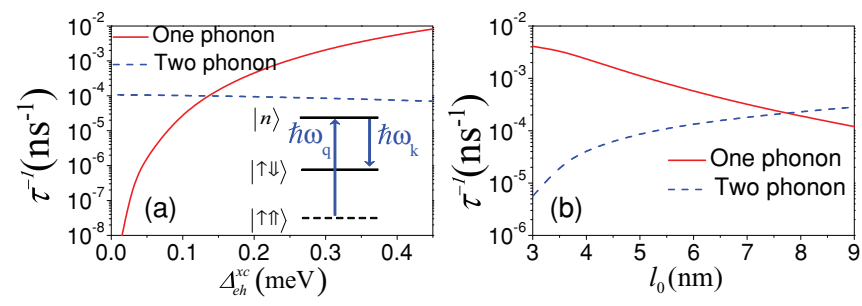

FIG. 2. (Color online) Exciton spin relaxation rates due to one- and two-phonon processes as a function of the $e-h$ exchange interaction (a) and the QD size (b) at $T=15 \mathrm{~K}$. In panel (a), $l_{0}=5$ $\mathrm{nm}$. In panel (b), size-dependent $\Delta_{e h}^{x c}$ is taken. The inset in panel (a) is a schematic of the two-phonon process.

Dresselhaus SOC, and the rates are calculated numerically using a second-order Fermi golden rule:

$$
\begin{aligned}
\tau_{h-D(2)}^{-1}= & \frac{2 \pi}{\hbar} \sum_{f} \sum_{\nu \mathbf{q}, \mathbf{k}}\left|\sum_{n} \frac{\left\langle f\left|V_{\nu \mathbf{k}}^{h}\right| n\right\rangle\left\langle n\left|V_{\nu \mathbf{q}}^{h}\right| i\right\rangle}{\left(E_{n}-E_{i}-E_{q}\right)}\right|^{2} \\
& \times \delta\left(E_{f i}+\hbar \omega_{\nu k}-\hbar \omega_{\nu q}\right) N_{q_{v}}\left(N_{k_{v}}+1\right) .
\end{aligned}
$$

Figure 2(a) compares the relaxation rates obtained with oneand two-phonon processes as a function of $\Delta_{e h}^{x c}$. One can see that single-phonon processes become rapidly inefficient for small $\Delta_{e h}^{x c}$, which is due to the decreasing density of phonon states. By contrast, two-phonon rates remain roughly constant. This is because there is no resonance condition for the transition to the virtual state. As a result, even if $\Delta_{e h}^{x c}$ is small, two-phonon processes may rely on the absorption and emission of energetic phonons, as long as the sum of their energies matches $E_{f}-E_{i}$. The insensitivity of two-phonon processes to $\Delta_{e h}^{x c}$ is analogous to that of individual holes to external magnetic fields, ${ }^{19}$ except that here the role of the magnetic field is played by the inherent exciton $e-h$ interaction.

Figure 2(a) shows that, for excitons, two-phonon process dominate over single-phonon ones only if $\Delta_{e h}^{x c}$ is small. To test if this is actually the case in self-assembled QDs, in Fig. 2(b) we compare one- and two-phonon rates as a function of the dot size, considering the size dependence of $\Delta_{e h}^{x c}$. Clearly, one-phonon processes dominate up to very large dot sizes, where $\Delta_{e h}^{x c}$ becomes small enough. It is worth noting that one-phonon processes dominate despite the moderately large temperature, $T=15 \mathrm{~K}$. This is because the $e$ - $h$ exchange acts as a fairly strong effective magnetic field. In what follows, we present an analysis for the exciton spin relaxation rates in main single-phonon processes to provide more understanding of the numerical data.

\section{ANALYSIS}

\section{A. Exciton wave functions}

For analysis, we begin with the energy spectrum of an exciton confined in a QD subject to relatively weak SOCs. Under the condition, the spin-mixed DX states as possible initial states can be expanded in the reduced basis formed by a few relevant exciton configurations and approximately 
expressed as

$$
\begin{aligned}
|X ; i\rangle \equiv & |\mathrm{DX}\rangle \\
& \times \propto\left(\left|\psi_{s}^{e} \uparrow ; \psi_{s}^{h} \Uparrow\right\rangle-C_{e-R}^{\mathrm{DX}}\left|\psi_{p+}^{e} \downarrow ; \psi_{s}^{h} \Uparrow\right\rangle\right. \\
& +i C_{e-D}^{\mathrm{DX}}\left|\psi_{p-}^{e} \downarrow ; \psi_{s}^{h} \Uparrow\right\rangle+i C_{h-\operatorname{lin}}^{\mathrm{DX}}\left|\psi_{s}^{e} \uparrow ; \psi_{p+}^{h} \Downarrow\right\rangle \\
& \left.+i C_{h-D}^{\mathrm{DX}}\left|\psi_{s}^{e} \uparrow ; \psi_{p+}^{h} \Downarrow\right\rangle\right), \\
\left|X ; i^{\prime}\right\rangle \equiv & \left|\mathrm{DX}^{\prime}\right\rangle \\
& \times \propto\left(\left|\psi_{s}^{e} \downarrow ; \psi_{s}^{h} \Downarrow\right\rangle+C_{e-R}^{\mathrm{DX}^{\prime}}\left|\psi_{p-}^{e} \uparrow ; \psi_{s}^{h} \Downarrow\right\rangle\right. \\
& +i C_{e-D}^{\mathrm{DX}}\left|\psi_{p+}^{e} \uparrow ; \psi_{s}^{h} \Downarrow\right\rangle+i C_{h-l^{\prime}}^{\mathrm{DX}^{\prime}}\left|\psi_{s}^{e} \downarrow ; \psi_{p-}^{h} \Uparrow\right\rangle \\
& \left.+i C_{h-D}^{\mathrm{DX}^{\prime}}\left|\psi_{s}^{e} \downarrow ; \psi_{p-}^{h} \Uparrow\right\rangle\right),
\end{aligned}
$$

where $\left|\psi_{i_{e}} \sigma_{e} ; \psi_{i_{h}} \sigma_{h}\right\rangle$ denotes the single-exciton configuration where an electron (a valence hole) occupies the $i_{e}$ th $\left(i_{h}\right.$ th) Fock-Darwin orbital with spin $\sigma_{e}=\uparrow / \downarrow\left(\sigma_{h}=\Uparrow\right.$ $/ \Downarrow)$, and $C_{j \text {-SO }}^{\xi}(\in R)$ are the (real) coefficients of the coupled configurations of dark exciton $\left(\xi=\mathrm{DX}, \mathrm{DX}^{\prime}\right)$ arising from the $j$-SO couplings $(j-\mathrm{SO}=e-R, e-D, h$-lin, $h-D)$. For brevity, the normalization constants are not shown in Eqs. (10) and (11).

Likewise, the spin-mixed bright-exciton ( $\left.\xi=\mathrm{BX}, \mathrm{BX}^{\prime}\right)$ states, as possible final states in Eq. (8), are written as

$$
\begin{aligned}
|X ; f\rangle \equiv & |\mathrm{BX}\rangle \\
& \times \propto\left(\left|\psi_{s}^{e} \uparrow ; \psi_{s}^{h} \Downarrow\right\rangle-C_{e-R}^{\mathrm{BX}}\left|\psi_{p+}^{e} \downarrow ; \psi_{s}^{h} \Downarrow\right\rangle\right. \\
& +i C_{e-D}^{\mathrm{BX}}\left|\psi_{p-}^{e} \downarrow ; \psi_{s}^{h} \Downarrow\right\rangle+i C_{h-\operatorname{lin}}^{\mathrm{BX}}\left|\psi_{s}^{e} \uparrow ; \psi_{p-}^{h} \Uparrow\right\rangle \\
& \left.+i C_{h-D}^{\mathrm{BX}}\left|\psi_{s}^{e} \uparrow ; \psi_{p-}^{h} \Uparrow\right\rangle\right), \\
\left|X ; f^{\prime}\right\rangle \equiv & \left|\mathrm{BX}^{\prime}\right\rangle \\
& \times \propto\left(\left|\psi_{s}^{e} \downarrow ; \psi_{s}^{h} \Uparrow\right\rangle+C_{e-R}^{\mathrm{BX}^{\prime}}\left|\psi_{p-}^{e} \uparrow ; \psi_{s}^{h} \Uparrow\right\rangle\right. \\
& +i C_{e-D}^{\mathrm{BX}}\left|\psi_{p+}^{e} \uparrow ; \psi_{s}^{h} \Uparrow\right\rangle+i C_{h-\operatorname{lin}}^{\mathrm{BX}}\left|\psi_{s}^{e} \downarrow ; \psi_{p+}^{h} \Downarrow\right\rangle \\
& \left.+i C_{h-D}^{\mathrm{BX}}\left|\psi_{s}^{e} \downarrow ; \psi_{p+}^{h} \Downarrow\right\rangle\right) .
\end{aligned}
$$

Figure 3 (4) depicts the schematics of the main low-lying exciton configurations for a dark-exciton (bright-exciton) state. By treating each SOC term separately and perturbatively

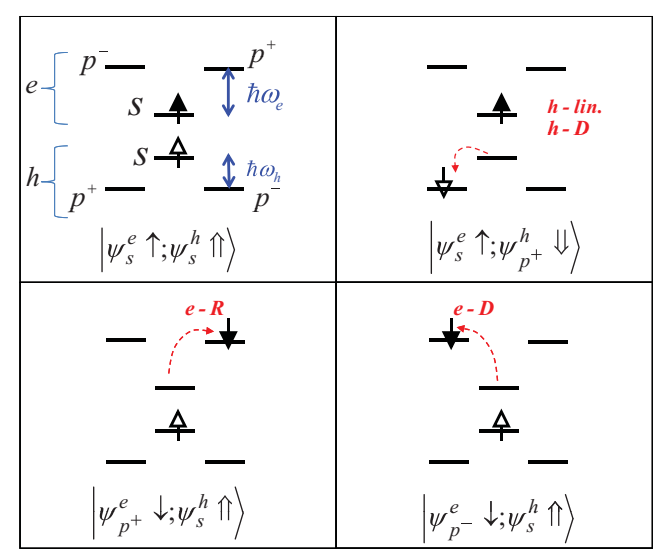

FIG. 3. (Color online) Schematics of the main low-lying exciton configurations coupled by various spin-orbital couplings for the darkexciton state $|\mathrm{DX}\rangle$ defined by Eq. (10).

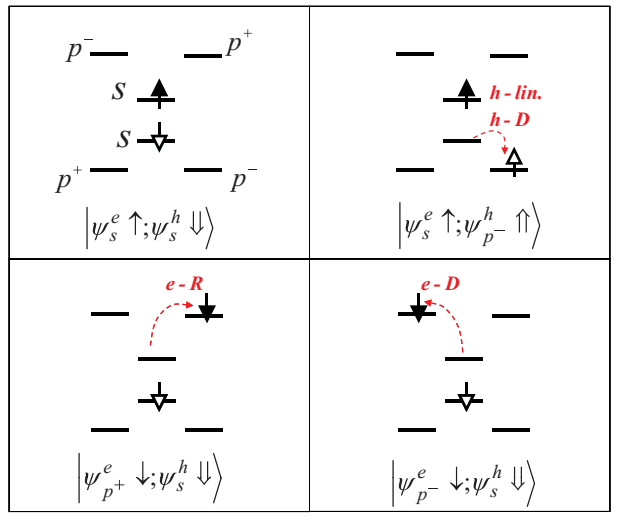

FIG. 4. (Color online) Schematics of the main low-lying exciton configurations coupled by various SOCs for the bright-exciton state $|\mathrm{BX}\rangle$ defined by Eq. (12).

in Eq. (10), one can derive the explicit expressions for the configuration coefficients $C_{j \text {-SO }}^{\xi}$ in terms of SOC constants $\left(\alpha_{j}, \beta_{j}\right)$, characteristic length of wave function extent $l_{0}$, energy quantization of QD $\varepsilon_{s p}^{j} \equiv \varepsilon_{p^{ \pm}}^{j}-\varepsilon_{s}^{j}$, and $\mathrm{BX}-\mathrm{DX}$ splitting $\Delta_{e h}^{x c}$. We assume that, prior to the inclusion of SOC effects, $s$-shell DX and BX states are split by $\Delta_{e h}^{x c}$. Then, the $p$-shell DX-BX splitting is $\Delta_{e h}^{x c} / 2$. The smaller splitting follows from the reduced orbital overlap in the short-range electron-hole exchange integral, $\int d^{3} r\left|\Psi_{p, 0}^{e}(\mathbf{r}) \Psi_{s, 0}^{h}(\mathbf{r})\right|^{2} .^{14}$

Higher shells (used in numerical calculations) are considered to have no sizable DX-BX splitting. The coefficients $C_{j-S O}^{\xi}$ derived under these conditions are summarized in Table II.

\section{B. Characteristic rate of spin relaxation}

Substituting Eqs. (10)-(13) into Eq. (8), the total spin relaxation rate in the transition from a $\mathrm{DX}$ state to $\mathrm{BX}$ ones can be decomposed into the individual rates arising from each SOC mechanism,

$$
\begin{aligned}
\tau_{j-\mathrm{SO}}^{-1}= & \frac{2 \pi}{\hbar} P_{j-\mathrm{SO}}^{2} \sum_{\nu \mathbf{q}}\left|M_{\nu}^{j}(\mathbf{q})\right|^{2}\left|\left\langle\Psi_{p, 0}\left|e^{i \mathbf{q} \cdot \mathbf{r}_{j}}\right| \Psi_{s, 0}\right\rangle\right|^{2} \\
& \times \delta\left(\Delta_{e h}^{x c}-\hbar \omega_{\nu q}\right) N_{q_{\nu}} .
\end{aligned}
$$

TABLE II. Explicit expressions for configuration coefficients of spin-mixed BX and DX states in terms of SOC constants $\left(\alpha_{j}, \beta_{j}\right)$, characteristic length of wave function extent $l_{0}$, energy quantization of QD $\varepsilon_{s p}^{j} \equiv \varepsilon_{p^{ \pm}}^{j}-\varepsilon_{\mathrm{s}}^{j}$, and BX-DX splitting $\Delta_{e h}^{x c}$. The formulations are derived by treating separatively and perturbatively each SOC mechanism. The parameters used in the calculations are summarized in Table V.

\begin{tabular}{lcccc}
\hline \hline$\xi$ & $C_{e-R}^{\xi}$ & $C_{e-D}^{\xi}$ & $C_{h-\operatorname{lin}}^{\xi}$ & $C_{h-D}^{\xi}$ \\
\hline$D X, D X^{\prime}$ & $\frac{\alpha_{e} / l_{0}}{\varepsilon_{s p}^{e}+3 \Delta_{e h}^{x c} / 4}$ & $\frac{\beta_{e} / l_{0}}{\varepsilon_{s p}^{e}+3 \Delta_{e h}^{x c} / 4}$ & $\frac{\alpha_{h} / l_{0}}{\varepsilon_{s p}^{h}+3 \Delta_{e h}^{x c} / 4}$ & $\frac{2 \beta_{h} / l_{0}^{3}}{\varepsilon_{s p}^{h}+3 \Delta_{e h}^{x c} / 4}$ \\
$B X, B X^{\prime}$ & $\frac{\alpha_{e} / l_{0}}{\varepsilon_{s p}^{e}-3 \Delta_{e h}^{x c} / 4}$ & $\frac{\beta_{e} / l_{0}}{\varepsilon_{s p}^{e}-3 \Delta_{e h}^{x c} / 4}$ & $\frac{\alpha_{h} / l_{0}}{\varepsilon_{s p}^{h}-3 \Delta_{e h}^{x c} / 4}$ & $\frac{2 \beta_{h} / l_{0}^{3}}{\varepsilon_{s p}^{h}-3 \Delta_{e h}^{x c} / 4}$ \\
\hline \hline
\end{tabular}


TABLE III. Explicit expressions for the spin-mixture factors

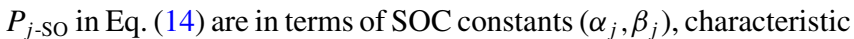
length of wave function extent $l_{0}$, energy quantization of QD $\varepsilon_{\mathrm{sp}}$, and BX-DX spin splitting $\Delta_{e h}^{x c}$.

\begin{tabular}{ccccc}
\hline \hline$P_{j-S O}$ & $P_{e-R}$ & $P_{e-D}$ & $P_{h-l i n}$ & $P_{h-D}$ \\
\hline & $\frac{3 \alpha_{e} / l_{0} \times \Delta_{e h}^{x c}}{2\left(\varepsilon_{s p}^{e}\right)^{2}}$ & $\frac{3 \beta_{e} / l_{0} \times \Delta_{e h}^{x c}}{2\left(\varepsilon_{s p}^{e}\right)^{2}}$ & $\frac{3 \alpha_{h} / l_{0} \times \Delta_{e h}^{x c}}{2\left(\varepsilon_{s p}^{h}\right)^{2}}$ & $\frac{3 \beta_{h} / l_{0}^{3} \times \Delta_{e h}^{x c}}{\left(\varepsilon_{s p}^{h}\right)^{2}}$ \\
\hline
\end{tabular}

The form factors $P_{j \text {-So }}$ are yielded by the slight spin admixture between DX states and BX states and, under the condition of weak SOC, are derived as

$$
\begin{aligned}
P_{e-R} & \approx\left|C_{e-R}^{\mathrm{BX}}-C_{e-R}^{\mathrm{DX}}\right|, \\
P_{e-D} & \approx\left|C_{e-D}^{\mathrm{BX}}-C_{e-D}^{\mathrm{DX}}\right|, \\
P_{h-\mathrm{lin}} & \approx\left|C_{h-\operatorname{lin}}^{\mathrm{BX}}-C_{h-\mathrm{lin}}^{\mathrm{DX}}\right|, \\
P_{h-D} & \approx\left|C_{h-D}^{\mathrm{BX}}-C_{h-D}^{\mathrm{DX}}\right| .
\end{aligned}
$$

Note in Eqs. (15)-(18) that it is the asymmetry of the spin admixture of DX and BX states that makes transitions between them feasible. Substituting the formulations for $C_{j \text {-So }}^{\xi}$ in Table II into the above equations for $P_{j-\mathrm{SO}}$, the spin-mixture factors $P_{j \text {-SO }}$ are expressed as

$$
P_{j-\mathrm{SO}}=3 \Lambda_{j-\mathrm{SO}} \Delta_{e h}^{x c} / 2\left(\varepsilon_{s p}^{j}\right)^{2},
$$

in terms of the characteristic energies of SOCs defined as $\Lambda_{e-R} \equiv \alpha_{e} / l_{0}, \Lambda_{e-D} \equiv \beta_{e} / l_{0}, \Lambda_{h-\text { lin }} \equiv \alpha_{h} / l_{0}$, and $\Lambda_{h-D} \equiv$ $2 \beta_{h} / l_{0}^{3}$, energy quantization of QD $\left(\varepsilon_{s p}^{j}\right)$, and the BX-DX spin splitting $\left(\Delta_{e h}^{x c}\right)$, as summarized in Table III. The expressions account for the fact that the probability of the DX-to-BX spin-state transition directly depends on the relative strength of the involved SOCs, the BX-DX spin splitting, and the energy quantization of the QD.

The spin relaxation rate for $j$-SO coupling can be reformulated as

$$
\tau_{j \text {-SO }}^{-1}=\frac{2 \pi}{\hbar} P_{j \text {-SO }}^{2} \sum_{\nu}\left|\bar{M}_{\nu}^{j}\left(q_{\nu 0}\right)\right|^{2} \rho_{\nu}\left(q_{\nu 0}\right) F_{\nu}\left(l_{0}, q_{\nu 0}\right) N_{q_{\nu 0}},
$$

where $q_{\nu 0}=\Delta_{e h}^{x c} /\left(\hbar c_{\nu}\right)$ is the magnitude of the wave vectors, $\rho_{\nu}=\frac{\Omega}{2 \pi^{2}} \frac{q_{v 0}^{2}}{\hbar c_{v}} \propto \Delta_{e h}^{x c 2}$ is the density of states of the resonant phonons involved in the relaxation process
( $\Omega$ denotes the crystal volume), and $\bar{M}_{v}^{j}(q)$ is the mean q-anisotropic phonon scattering matrix element, separated from the angular part $I_{v}\left(\theta_{q}, \phi_{q}\right)$ in the matrix element $M_{v}^{j}(\mathbf{q}) \equiv \bar{M}_{v}^{j}(q) I_{v}\left(\theta_{q}, \phi_{q}\right)$. The function $F_{v}\left(l_{0}, q\right) \equiv$ $(1 / 4 \pi) \int_{0}^{2 \pi} \int_{0}^{\pi}\left|I_{\nu}\left(\theta_{q}, \phi_{q}\right)\right|^{2}\left|\left\langle\Psi_{p, 0}\left|e^{i \mathbf{q} \cdot \mathbf{r}}\right| \Psi_{s, 0}\right\rangle\right|^{2} \sin \theta_{q} d \theta_{q} d \phi_{q}$ considers the anisotropy of phonon coupling and the localization of the particle wave function in the QD. Table IV summarizes the expressions for $\bar{M}_{v}^{j}, I_{\nu}, \rho_{\nu}$, and $F_{v}$ as functions of the wave vector of resonant phonons $\mathbf{q}_{\nu 0}=\left(q_{\nu 0}, \theta_{q_{\nu 0}}, \phi_{q_{v 0}}\right)$ and/or the characteristic length of the wave function extent $l_{0}$ defined by the 2D parabolic model.

Since $\left|\bar{M}_{T P}\right|^{2} \propto 1 / q_{T P 0}$, while $\left|\bar{M}_{L D(T D)}\right|^{2} \propto q_{L D(T D) 0}$, transversal piezoelectric phonon interaction is dominant in transitions between $\mathrm{BX}$ and DX states, which in selfassembled dots are split by only $\sim 10^{2} \mu \mathrm{eV} .{ }^{17}$ Thus, the total coupling strength of phonons involved in a spin relaxation can be estimated by $\left|\bar{M}_{T P}\right|^{2} \rho_{T P} \propto q_{T P 0} \propto \Delta_{e h}^{x c}$, a product of the dominant transversal piezoelectric phonon coupling and the phonon density of states.

The numerical results of Fig. 1 show that the Dresselhaus SOCs are generally dominant over other possible spinadmixture mechanisms in exciton spin relaxation. Among all $p$-linear terms, the $e$-Dresselhaus SOC leads to faster relaxation rates than other Rashba terms since $\beta_{e} \gg \alpha_{e}, \alpha_{h}$ (see Table V). Yet, the cubic $h$-Dresselhaus SOC plays the main role because of the heavier mass of the hole and the strong confinement of the dot, as shown by the analysis below.

Taking the fact that $l_{0} q_{T P 0} \ll 1$ and $N_{q_{v 0}} \approx k_{B} T / \Delta_{e h}^{x c}$ (since $k_{B} T \gg \Delta_{e h}^{x c}$ ), the characteristic spin relaxation rates of excitons in a QD via the main $e$ - and $h$-Dresselhaus SOCs are derived as

$$
\begin{gathered}
\tau_{e}^{-1} \approx K \beta_{e}^{2} m_{e}^{4}\left(\Delta_{e h}^{x c}\right)^{4} l_{0}^{8} T, \\
\tau_{h}^{-1} \approx K\left(2 \beta_{h}\right)^{2} m_{h}^{4}\left(\Delta_{e h}^{x c}\right)^{4} l_{0}^{4} T,
\end{gathered}
$$

respectively, where $K \equiv \frac{48 \pi m_{0}^{4} e^{2} h_{14}^{2} k_{B}}{35 \hbar^{9} \epsilon^{2} d c_{T P}^{2}}$ is a constant. The domi-

\begin{tabular}{|c|c|c|c|c|}
\hline & $v=L D$ & $v=T D$ & $v=L P$ & $v=T P$ \\
\hline$\left|\bar{M}_{v}^{j}\left(q_{v}\right)\right|^{2}$ & $\frac{\hbar D_{j, L D}^{2}}{2 d c_{L D} \Omega} q_{v}$ & $\frac{\hbar D_{j, T D}^{2}}{15 d c_{T D} \Omega} q_{v}$ & $\frac{96 \pi^{2} \hbar e^{2} h_{14}^{2}}{35 \epsilon^{2} d c_{L P} \Omega} \frac{1}{q_{\nu}}$ & $\frac{128 \pi^{2} \hbar e^{2} h_{14}^{2}}{35 \epsilon^{2} d c_{T P} \Omega} \frac{1}{q_{\nu}}$ \\
\hline$\left|I_{v}\left(\theta_{q_{v}}, \phi_{q_{v}}\right)\right|^{2}$ & 1 & $\frac{15}{4} \sin ^{2} 2 \theta_{q_{v}}$ & $\frac{105}{4}\left(\sin ^{2} \theta_{q_{v}} \cos \theta_{q_{v}} \sin 2 \phi_{q_{v}}\right)^{2}$ & $\begin{array}{c}\frac{35}{16}\left(\sin ^{4} \theta_{q_{\nu}} \sin ^{2} 2 \phi_{q_{\nu}}+\sin ^{2} 2 \theta_{q_{\nu}}\right) \\
-\frac{315}{16}\left(\sin ^{2} \theta_{q_{\nu}} \cos \theta_{q_{\nu}} \sin 2 \phi_{q_{\nu}}\right)^{2}\end{array}$ \\
\hline$\rho_{v}\left(q_{v}\right)$ & $\frac{\Omega}{8 \pi^{3}} \frac{4 \pi q_{v}^{2}}{\hbar c_{\nu}}$ & $\frac{\Omega}{8 \pi^{3}} \frac{4 \pi q_{v}^{2}}{\hbar c_{v}}$ & $\frac{\Omega}{8 \pi^{3}} \frac{4 \pi q_{v}^{2}}{\hbar c_{v}}$ & $\frac{\Omega}{8 \pi^{3}} \frac{4 \pi q_{v}^{2}}{\hbar c_{v}}$ \\
\hline$F_{v}\left(l_{0}, q_{v}\right)$ & $\frac{1}{6}\left(\frac{2}{5}+\frac{1}{l_{0}^{2} q_{v}^{2}}\right)^{-1}$ & $\frac{1}{7}\left(\frac{1}{3}+\frac{1}{l_{0}^{2} q_{v}^{2}}\right)^{-1}$ & $\frac{1}{6}\left(\frac{4}{11}+\frac{1}{l_{0}^{2} q_{v}^{2}}\right)^{-1}$ & $\frac{1}{6}\left(\frac{13}{33}+\frac{1}{l_{0}^{2} q_{v}^{2}}\right)^{-1}$ \\
\hline
\end{tabular}
nant role of $h-D$ SOC in the exciton spin relaxation is identified by the high ratio of $\tau_{e-D}$ to $\tau_{h-D}$,

$$
\frac{\tau_{e-D}}{\tau_{h-D}}=\left(\frac{2 \beta_{h} / l_{0}^{3}}{\beta_{e} / l_{0}}\right)^{2} \times \frac{m_{h}}{m_{e}} \gg 1,
$$

TABLE IV. The expressions for the functions $I_{v}, \bar{M}_{v}^{j}, \rho_{v}$, and $F$ in terms of phonon parameters for InGaAs, the wave vector of phonon $\mathbf{q}_{v}=\left(q_{v}, \theta_{q_{v}}, \phi_{q_{v}}\right)$ represented in the spherical coordinate, and the characteristic length of wave function extent $l_{0}$ defined within the $2 \mathrm{D}$ parabolic model. The symbols and values of the phonon parameters for InGaAs appearing in this table are summarized in Table V. 
which is explicitly shown to be much greater than 1 for InAs self-assembled QDs with typical $l_{0}<10 \mathrm{~nm}$. The above equations account for the faster exciton spin relaxation rate via $h$-SOC than via $e$-SOC due to the heavier mass of the hole, which results in the hole having a weaker quantization and a higher value of spin-mixture factor $P$, and a strong quantum confinement of the $\operatorname{dot}\left(\operatorname{small} l_{0}\right)$. For very large QDs, however, the $l_{0}$ dependence indicates that $e$-SOC will eventually become dominant. $^{15}$

Figure 5 (solid lines) shows the dominant form factors $P_{h-D}^{2}$, $\left|\bar{M}_{T P}\right|^{2} \rho_{T P}, F_{T P}$, and $N_{q_{T P}}$ as functions of $l_{0}$. It is clearly seen that, with reducing dot sizes, the three former functions make increasing contributions to the total spin relaxation rate. This is because the increased $\Delta_{e h}^{x c}$ and $q_{\nu 0}$ in small dots increase the spin admixture between the DX states and the BX ones, the number of involved phonons, and the strength of effective phonon coupling.

\section{Power-law dependencies}

If $\Delta_{e h}^{x c}$ is treated as a constant, Eqs. (21) and (22) predict decreased relaxation rates by reducing size, which is indeed the behavior of independent electrons and holes. ${ }^{2}$ Taking the size dependence of spin splitting $\left(\Delta_{e h}^{x c} \propto l_{0}^{-2}\right)$ into account, however, the power laws Eqs. (21) and (22) are reformulated as

$$
\begin{gathered}
\tau_{e}^{-1} \propto \beta_{e}^{2} \times m_{e}^{4} \times T, \\
\tau_{h}^{-1} \propto \beta_{h}^{2} \times m_{h}^{4} \times l_{0}^{-4} \times T .
\end{gathered}
$$
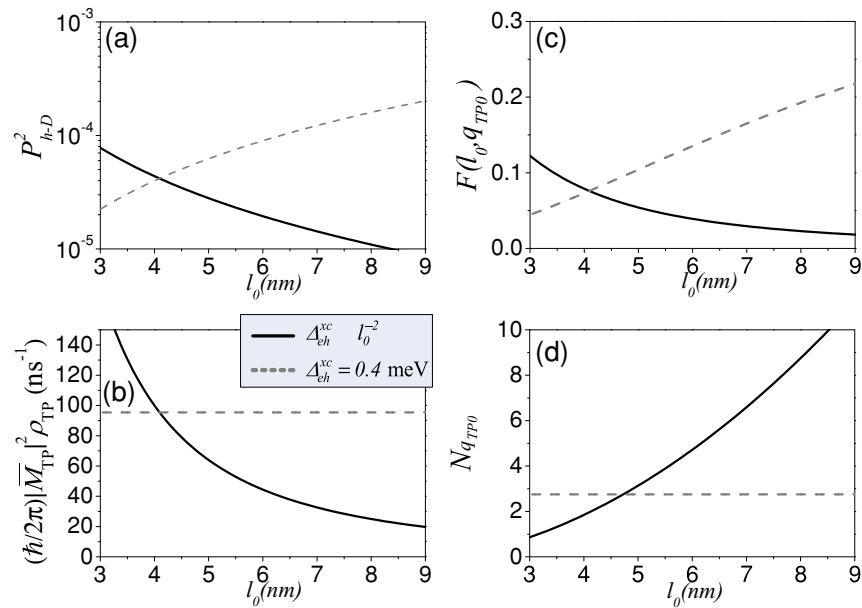

FIG. 5. (Color online) Calculated (a) spin-mixture function $P$, (b) product of mean phonon coupling and density of states of involved phonons $|\bar{M}|^{2} \rho$, (c) correction factor $F$, and (d) phonon population $N_{q_{T P 0}}$ as functions of $l_{0}$ for the dominant $h-D$ SOC and transversal piezoelectric phonon (TP) couplings. The product of the four quantities determine the main exciton spin relaxation rates of QDs as shown by Eq. (20). The results calculated with fixed $\Delta_{e h}^{x c}=0.4$ $\mathrm{meV}$ are indicated by dashed lines for comparison.

These power laws account for the features of roughly constant $\tau_{e-R / D}^{-1}$ and enhanced $\tau_{h-D}^{-1}$ by the reduced size of QDs observed in Fig. 1. To highlight the significance of the size dependence of the $e$ - $h$ exchange splitting, Figs. 1 and 5 also show the total spin relaxation rate calculated with a fixed $\Delta_{e h}^{x c}=0.4 \mathrm{meV}$

TABLE V. Summary of the parameters used in the analysis and numerical calculations for InGaAs quantum dots throughout this work. In some cases (marked with a superscript $*$ ) for which only the parameters for binary compounds (InAs or GaAs) are available, the parameters

\begin{tabular}{|c|c|c|c|}
\hline Parameter & Symbol & Value & Refs. \\
\hline Electron effective mass & $m_{e}$ & $0.05 m_{0}$ & $21 *$ \\
\hline Hole effective mass & $m_{h}$ & $0.2 m_{0}$ & $19 *$ \\
\hline Thickness of QD & $d_{z}$ & $3 \mathrm{~nm}$ & \\
\hline Bulk exciton Bohr radius & $a_{B}^{*}$ & $25 \mathrm{~nm}$ & $22 *$ \\
\hline Bulk $e$ - $h$ exchange energy & $\Delta_{e h, \text { bulk }}^{x c}$ & $4.3 \mu \mathrm{eV}$ & 9 \\
\hline$e$-Rashba coupling constant & $\alpha_{e}$ & $0.1 \mathrm{eV} \cdot \AA$ & 23 \\
\hline$e$-Dresselhaus coupling constant & $\beta_{e}$ & $0.65 \mathrm{eV} \cdot \AA$ & \\
\hline$h$-linear coupling constant & $\alpha_{h}$ & $11 \mathrm{meV} \cdot \AA$ & $12 *$ \\
\hline$h$-Dresselhaus coupling constant & $\beta_{h}$ & $190 \mathrm{eV} \cdot \AA^{3}$ & 15 \\
\hline Longitudinal sound velocity of acoustic phonon & $c_{L D / L P}$ & $4720 \mathrm{~m} / \mathrm{s}$ & 17 \\
\hline Transversal sound velocity of acoustic phonon & $c_{T D / T P}^{L D / P}$ & $3340 \mathrm{~m} / \mathrm{s}$ & 17 \\
\hline Density of material & $d$ & $5310 \mathrm{~kg} / \mathrm{m}^{3}$ & 17 \\
\hline Hydrostatic deformation potential constant for electron $\left(a_{c}\right)$ & $D_{e, L D}$ & $-7.17 \mathrm{eV}$ & 21 \\
\hline Hydrostatic deformation potential constant for hole $\left(a_{v}+b / 2\right)$ & $D_{h, L D}$ & $-2.16 \mathrm{eV}$ & 21 \\
\hline Uniaxial deformation potential constant for hole $(-3 b / 2)$ & $D_{h, T D}$ & $3 \mathrm{eV}$ & 21 \\
\hline Piezoelectric constant & $h_{14}$ & $1.41 \times 10^{9} \mathrm{~V} / \mathrm{m}$ & 17 \\
\hline Static dielectric constant & $\epsilon$ & 12.9 & 17 \\
\hline Dresselhaus constant & $\gamma_{D}$ & $100 \mathrm{eV} \cdot \AA^{3}$ & $15 *$ \\
\hline Split-off gap energy/(band gap energy + split-off gap energy) & $\eta$ & 0.35 & $15 *$ \\
\hline Heavy-hole and light-hole splitting & $\Delta_{h h}^{l h}$ & $0.15 \mathrm{eV}$ & $15 *$ \\
\hline Luttinger parameter & $\gamma_{2}$ & 4.2 & 21 \\
\hline Conduction band offset & $V_{c o}$ & $0.3 \mathrm{eV}$ & $21 *$ \\
\hline Valence band offset & $V_{v o}$ & $0.2 \mathrm{eV}$ & $21 *$ \\
\hline
\end{tabular}
for InGaAs are determined by taking interpolated values. 
(dashed lines). The obtained behavior is drastically different from that with size-dependent $\Delta_{e h}^{x c}$.

\section{SUMMARY}

In conclusion, we have calculated the relaxation rates between DX and BX states in InGaAs QDs for a wide number of spin-flip mechanisms and shown that hole-Dresselhaus SOC assisted by single-phonon processes is the dominant channel. The $e$ - $h$ exchange splitting acts as an internal magnetic field enhancing SOC mechanisms. Since the splitting grows with the confinement, the smaller the dot the faster the exciton spin relaxation. This is contrary to the well-known behavior of individual electrons or holes, for which relaxation is suppressed by the confinement.

\section{ACKNOWLEDGMENTS}

SJC is grateful to the National Science Council of Taiwan (Contract No. NSC-98-2112-M-009-011-MY2), the National Center of Theoretical Sciences and the National Center for
High-Performance Computing of Taiwan in Hsinchu for supporting. Support from MCINN Project CTQ2008-03344 and the Ramon y Cajal Program (JIC) is acknowledged.

\section{APPENDIX: PARAMETERS}

Table V summarizes the symbols and values of the parameters used in the analysis and the numerical calculations throughout this work. The determination of the Dresselhaus constant $\beta_{e}$ follows the formalism $\beta_{e}=\gamma_{D}\left\langle p_{z}^{2}\right\rangle$, where the bulk Dresselhaus SO constant $\gamma_{D}=100 \mathrm{eV} \cdot \AA^{3}$ is taken for InGaAs and the term $\left\langle p_{z}^{2}\right\rangle$ is evaluated by solving a onedimesional Schrödinger equation for a square well in the $z$ direction using a finite-difference method. ${ }^{20}$ The values of the conduction (valence) band offset $V_{c o}\left(V_{v o}\right)$ for the vertical square well is given in Table $\mathrm{V}$. The parameter $\beta_{h}$ is determined by the evaluation of $\beta_{h}=3 \gamma_{D} \gamma_{2}\left\langle p_{z}^{2}\right\rangle / 2 m_{0} \eta \Delta_{h h}^{l h}$, with $\gamma_{2}$ as the Luttinger parameter, $m_{0}$ as the free electron mass, $\Delta_{h h}^{l h}$ as the energy splitting between heavy holes and light holes, and the factor defined as $\eta=\Delta_{s o} /\left(E_{g}+\Delta_{s o}\right)$, where $\Delta_{s o}$ is the split-off gap energy and $E_{g}$ is the band gap energy for InGaAs (see Table V). ${ }^{15}$ *sjcheng@mail.nctu.edu.tw

${ }^{1}$ R. Hanson, L. P. Kouwenhoven, J. R. Petta, S. Tarucha, and L. M. K. Vandersypen, Rev. Mod. Phys. 79, 1217 (2007).

${ }^{2}$ A. V. Khaetskii and Y. V. Nazarov, Phys. Rev. B 61, 12639 (2000).

${ }^{3}$ S. Amasha, K. MacLean, I. P. Radu, D. M. Zumbühl, M. A. Kastner, M. P. Hanson, and A. C. Gossard, Phys. Rev. Lett. 100, 046803 (2008).

${ }^{4}$ J. R. Petta, A. C. Johnson, J. M. Taylor, E. A. Laird, A. Yacoby, M. D. Lukin, C. M. Marcus, M. P. Hanson, and A. C. Gossard, Science 309, 2180 (2005).

${ }^{5}$ A. J. Shields, Nat. Photonics 1, 215 (2007).

${ }^{6}$ S. Strauf, N. G. Stoltz, M. T. Rakher, L. A. Coldren, P. M. Petroff, and D. Bouwmeester, Nat. Photonics 1, 704 (2007).

${ }^{7}$ M. Reischle, G. J. Beirne, R. Rossbach, M. Jetter, and P. Michler, Phys. Rev. Lett. 101, 146402 (2008).

${ }^{8}$ J. Johansen, B. Julsgaard, S. Stobbe, J. M. Hvam, and P. Lodahl, Phys. Rev. B 81, 081304 (R) (2010).

${ }^{9}$ M. Bayer, G. Ortner, O. Stern, A. Kuther, A. A. Gorbunov, A. Forchel, P. Hawrylak, S. Fafard, K. Hinzer, T. L. Reinecke, S. N. Walck, J. P. Reithmaier, F. Klopf, and F. Schäfer, Phys. Rev. B 65, 195315 (2002).

${ }^{10}$ E. Tsitsishvili and H. Kalt, Phys. Rev. B 82, 195315 (2010).
${ }^{11}$ K. Roszak, V. M. Axt, T. Kuhn, and P. Machnikowski, Phys. Rev. B 76, 195324 (2007); 77, 249905(E) (2008).

${ }^{12}$ E. Tsitsishvili, R. v. Baltz, and H. Kalt, Phys. Rev. B 72, 155333 (2005).

${ }^{13}$ P. Hawrylak, Phys. Rev. B 60, 5597 (1999).

${ }^{14}$ R. Romestain and G. Fishman, Phys. Rev. B 49, 1774 (1994).

${ }^{15}$ D. V. Bulaev and D. Loss, Phys. Rev. Lett. 95, 076805 (2005).

${ }^{16}$ S. I. Erlingsson and Y. V. Nazarov, Phys. Rev. B 66, 155327 (2002).

${ }^{17}$ J. I. Climente, A. Bertoni, G. Goldoni, and E. Molinari, Phys. Rev. B 74, 035313 (2006).

${ }^{18}$ T. Meunier, I. T. Vink, L. H. Willems van Beveren, K. J. Tielrooij, R. Hanson, F. H. L. Koppens, H. P. Tranitz, W. Wegscheider, L. P. Kouwenhoven, and L. M. K. Vandersypen, Phys. Rev. Lett. 98, 126601 (2007).

${ }^{19}$ M. Trif, P. Simon, and D. Loss, Phys. Rev. Lett. 103, 106601 (2009).

${ }^{20}$ J. I. Climente, A. Bertoni, G. Goldoni, M. Rontani, and E. Molinari, Phys. Rev. B 76, 085305 (2007).

${ }^{21}$ I. Vurgaftman, J. R. Meyer, and L. R. Ram-Mohan, J. Appl. Phys. 89, 5815 (2001).

${ }^{22}$ H. Fu, L.-W. Wang, and A. Zunger, Phys. Rev. B 59, 5568 (1999).

${ }^{23}$ G. Engels, J. Lange, T. Schapers, and H. Luth, Phys. Rev. B 55, R1958 (1997). 